\title{
Physical activity attenuates the mid-adolescent peak in insulin resistance but by late adolescence the effect is lost: a longitudinal study with annual measures from 9-16 years (EarlyBird 66)
}

\author{
Brad S. Metcalf ${ }^{1,2}$ • Joanne Hosking ${ }^{3}$ - William E. Henley ${ }^{1}$ - Alison N. Jeffery ${ }^{3}$. \\ Mohammod Mostazir ${ }^{1} \cdot$ Linda D. Voss ${ }^{3} \cdot$ Terence J. Wilkin $^{1}$
}

Received: 26 March 2015 / Accepted: 10 July 2015 / Published online: 12 August 2015

(C) Springer-Verlag Berlin Heidelberg 2015

\begin{abstract}
Aims/hypothesis The aim of this work was to test whether the mid-adolescent peak in insulin resistance (IR) and trends in other metabolic markers are influenced by long-term exposure to physical activity.

Methods Physical activity (7 day ActiGraph accelerometry), HOMA-IR and other metabolic markers (glucose, fasting insulin, $\mathrm{HbA}_{1 \mathrm{c}}$, lipids and $\mathrm{BP}$ ) were measured annually from age 9 years to 16 years in 300 children ( 151 boys) from the EarlyBird study in Plymouth, UK. The activity level of each child was characterised, with $95 \%$ reliability, by averaging their eight annual physical activity measures. Age-related trends in IR and metabolic health were analysed by multilevel modelling, with physical activity as the exposure measure (categorical and continuous) and body fat percentage (assessed by dual-energy X-ray absorptiometry) and pubertal status (according to age at peak height velocity and Tanner stage) as covariates.

Results The peak in IR at age 12-13 years was $17 \%$ lower $(p<0.001)$ in the more active adolescents independently of body fat percentage and pubertal status. However, this difference diminished progressively over the next 3 years and had disappeared completely by the age of 16 years (e.g. difference
\end{abstract}

Brad S. Metcalf

b.metcalf@exeter.ac.uk

1 Institute of Health Research, University of Exeter Medical School, Exeter, UK

2 Sport and Health Sciences, College of Life and Environmental Sciences, University of Exeter, St Luke's Campus, Heavitree Road, Exeter EX1 2LU, UK

3 Department of Endocrinology and Metabolism, Plymouth University Peninsula Schools of Medicine and Dentistry, Plymouth, UK was $-14 \%$ at 14 years, $-8 \%$ at 15 years and $+1 \%$ at 16 years; 'physical activity $\times \mathrm{age}^{2}$ ' interaction, $\left.p<0.01\right)$. Triacylglycerol levels in girls $(-9.7 \%, p=0.05)$ and diastolic blood pressure in boys $(-1.20 \mathrm{mmHg}, p=0.03)$ tended to be lower throughout adolescence in the more active group.

Conclusions/interpretation Our finding that physical activity attenuates IR during mid-adolescence may be clinically important. It remains to be established whether the temporary attenuation in IR during this period has implications for the development of diabetes in adolescence and for future metabolic health generally.

Keywords Adolescence $\cdot$ Children $\cdot$ Exercise $\cdot$ Insulin resistance $\cdot$ Metabolic health $\cdot$ Physical activity $\cdot$ Teenagers

$\begin{array}{ll}\text { Abbreviations } \\ \text { DBP } & \text { Diastolic BP } \\ \text { DXA } & \text { Dual-energy X-ray absorptiometry } \\ \text { IR } & \text { Insulin resistance } \\ \text { MAP } & \text { Mean arterial pressure } \\ \text { MET } & \text { Metabolic equivalent } \\ \text { SBP } & \text { Systolic BP }\end{array}$

\section{Introduction}

The prevalence of childhood obesity has increased in much of the industrialised world over the past two decades with one-insix children in the UK [1] and USA [2] currently being obese. This is of concern because obesity appears to underlie much of the insulin resistance (IR) that leads to diabetes, cardiovascular disease and the metabolic syndrome [3]. The cause of obesity is multifactorial but ultimately results from a chronic imbalance between energy intake and energy expenditure. 
Physical activity accounts for around $35-45 \%$ of energy expenditure in children and adolescents [4] and, being potentially modifiable, it is often incorporated intuitively into programmes designed to reduce childhood obesity.

Systematic reviews have shown that activity interventions in children lead to only small improvements in body mass and body fat [5-7]. However, other reviews have shown that activity interventions (especially those of at least moderateintensity activity) consistently reduce IR and improve metabolic health, even when body fat is unchanged [8,9]. Together, these findings suggest that physical activity might improve IR and metabolic health via two mechanisms - one through the lowering of body fat, one independent of body fat. Observational studies showing an inverse association between physical activity and IR, even after controlling for body mass and body fat, support this conclusion [8]. The association tended to be found only in adolescent children, while the evidence in pre-adolescent children was not so clear [8]. Our own (EarlyBird) data show no association between activity and IR in pre-adolescence (age 8 years) [10]. The higher levels of IR in the adolescent age group that we and others have reported [11-13] may explain the emergence of an association with physical activity around that time.

IR appears to be a factor in both type 1 and type 2 diabetes [14]. An understanding of the metabolic response to physical activity at an age when the incidence of childhood diabetes peaks could be important in gauging the role of physical activity in diabetes prevention. Using annual recordings of objectively measured physical activity, body composition, insulin, glucose, lipids and blood pressure collected from a single cohort throughout the entire period of adolescence (age 916 years), we have sought to establish when, and to what extent, physical activity in contemporary children has an impact on IR and other markers of metabolic health.

\section{Methods}

\section{Design, setting and participants}

EarlyBird is a non-intervention prospective cohort study of 347 healthy children (174 boys, 173 girls) recruited from 53 primary schools in Plymouth, UK (307 children were recruited at age 5 years in 2000 and 2001 and 40 more at age 9 years in 2004 and 2005 to redress a sex imbalance). An initial sample size of 307 was calculated in order to deem even a small association $\left(r=0.16, R^{2}=2.5 \%\right.$ [15]) between physical activity and obesity-related health outcomes statistically significant at the $5 \%$ level $(p<0.01)$ with $80 \%$ power. Most $(98.6 \%, n=342)$ were of European descent and five children $(1.5 \%)$ were of mixed race, reflecting the racial mix of the city $(1.6 \%$ according to the 2001 census [16]). The cohort underwent measurements annually (time between visits: mean $=1.0$ year, SD
0.1 years). Data from pre-puberty (age $5-8$ years) have already been reported [10]; the present report covers the subsequent eight annual time points spanning all of adolescence from the age of 9 years to 16 years (early adolescence $\sim 9-11$ years, midadolescence $\sim 12-14$ years, late adolescence $\sim 15-16$ years). Written consent from the parent and assent from the child was obtained at each visit. Ethical approval was obtained from the Plymouth Local Research Ethics Committee (South and West Devon Health Authority) in 1999 and updated regularly. The study's rationale, recruitment procedures and protocol have been reported in detail elsewhere [17].

\section{Main outcome and exposure measures}

Physical activity Activity was measured objectively using ActiGraph accelerometers (Model 7164 - formerly MTI/CSA; ActiGraph, Fort Walton Beach, FL, USA). The particular model that we used offers good technical reproducibility [18] and its data correlate well with those of free-living activity-related energy expenditure obtained using indirect calorimetry $[19,20]$. The accelerometers were worn around the child's waist and were set to run continuously for 7 days at each annual time point. To eliminate any acute effect that physical activity may have on the measurement of metabolic health, the accelerometers were worn during the week that followed the measurements. The children were asked to wear the accelerometer every day from the time they got out of bed in the morning to the time they went to bed at night, removing it only for any water-based activity (e.g. swimming, bathing, showering, etc.). Only recordings that captured at least 4 days monitoring (incorporating at least $9 \mathrm{~h}$ wear time and including at least one weekend day) were included in the analyses as four is the minimum number of days required to achieve $70 \%$ reliability [21]. The ActiGraph 7164 records change in vertical acceleration (expressed as 'counts') 600 times per minute, which for the present study was integrated into $1 \mathrm{~min}$ epochs. Two components of physical activity were adduced from the ActiGraph data at each annual time point: time spent in moderate-and-vigorous-intensity activity (expressed as $\mathrm{min} /$ day) and total volume of activity (expressed as counts/min). Moderate-and-vigorous physical activity was the measure of physical activity used in the main analysis as the primary exposure measure, while total volume of activity was used only in the sensitivity analysis. The recommended 'counts/ min' threshold varies widely for the term 'moderate-and-vigorous' activity [22]; for this study the threshold was specific to 3 metabolic equivalents (METs), deemed by the UK government to be the lower limit of moderate activity [23]. Two studies that measured energy expenditure and ActiGraph counts simultaneously in children have shown that 3 METs is equivalent to $\sim 2,500$ ActiGraph counts/min $[19,20]$. This cut-point is very similar to the one that was reported by Trost et al as being the most appropriate of the five published cut- 
points for 'moderate-and-vigorous'-intensity activity [24]. The children and their parents were asked to record periods when the child removed the accelerometer during waking time, so that false periods of inactivity could be identified. The sensitivity of each accelerometer was measured by a motorised turntable [18]. Seasonality was measured by the number of relevant daylight hours (between 08:00 hours and 22:00 hours) specific to Plymouth, UK for the week the accelerometer was worn [25].

Each measure of physical activity was adjusted to the mean number of daylight hours $(10.5 \mathrm{~h} /$ day $)$ and to the mean ActiGraph sensitivity. Reported periods of non-compliance and intervals of zero accelerometer counts lasting 17 consecutive minutes or more (assumed to be unreported noncompliance) were replaced with the mean accelerometer counts recorded at the same clock time on the other week or weekend days. The 17 min threshold is based on an unpublished calibration study of 30 children that we (B. S. Metcalf and T. J. Wilkin) carried out and is between the $20 \mathrm{~min}$ threshold recommended in young children [26] and the $10 \mathrm{~min}$ threshold that others have applied to similar-aged children [27].

Metabolic variables A venous blood sample was taken at 09:00 hours after the children had fasted overnight. Serum insulin was measured using an Immulite analyser (Diagnostic Products Corporation, Los Angeles, CA, USA). The crossreactivity with proinsulin was $<1 \%$, the inter-assay $\mathrm{CV}$ was $\sim 9 \%$ and the detection limit of the assay was $2.0 \mathrm{mU} / 1$ ( $\sim 14 \mathrm{pmol} / \mathrm{l})$. Glucose was measured using a Cobas Integra 700 analyser (Roche Diagnostics, Burgess Hill, UK), with inter-assay CV being $\sim 2 \%$. The values for insulin and glucose were used to derive a measure of IR using the HOMA-IR program [28], downloaded from the University of Oxford Diabetes Trials Unit website (www.dtu.ox.ac.uk). HOMA-IR has been validated against the euglycaemic clamp $(r=0.91)$ in healthy youths [29]. $\mathrm{HbA}_{1 \mathrm{c}}$ levels were measured by automated high-performance liquid chromatography using a Menarini Biomen HA 8140 analyser (Menarini, High Wycombe, UK). Triacylglycerol, cholesterol and HDL-cholesterol were measured on a Cobas Integra 700 analyser; LDL-cholesterol and the cholesterol/HDL ratio were derived accordingly. Systolic and diastolic BP (SBP and DBP, respectively) was taken with a semi-automated sphygmomanometer and the mean arterial pressure $(\mathrm{MAP})$ was calculated as $(\mathrm{SBP}+[2 \times \mathrm{DBP}]) / 3$.

Covariates Each year, the percentage body fat of the participants was measured from a whole-body dual-energy X-ray absorptiometry (DXA) scan performed with a Prodigy Advance fan beam densitometer (GE Healthcare, Little Chalfont, UK). Pubertal status was accounted for with two measures - age at peak height velocity (calculated from sixmonthly measurements of height) and pubic hair development (self-reported using the Tanner stage 1-5 drawings where $1=$ no pubic hair).

\section{Statistical analysis}

The overall long-term physical activity exposure level of each child was characterised by averaging their moderate-andvigorous activity measures across all eight annual time points. In doing so, the children were positioned/ranked according to how active they had been throughout the entire adolescent period with $95 \%$ reliability (compared with just $70 \%$ reliability achieved with one annual measure of physical activity). As moderate-and-vigorous activity changed little from the age of 9 years to 16 years, averaging was carried out on $\min /$ day data, but for total volume of activity, which declined during adolescence, the data were standardised into age- and sexspecific $z$ scores before being averaged. The average moderate-and-vigorous activity level was analysed both as a continuous variable and, for ease of presentation and interpretation, as a categorical variable (those above the sex-specific median [boys $50 \mathrm{~min} /$ day; girls $35 \mathrm{~min} /$ day] were labelled as 'more active' and those below as 'less active').

Multi-level modelling is ideal for the analyses of longitudinal/repeated measures data and was therefore used in this study to determine whether the age-related changes in metabolic health differed depending on the overall activeness of the child. Multi-level modelling produces more robust model coefficients than standard cross-sectional techniques as it accounts for between-child variation by modelling within-child trajectories. This is achieved by entering age (and any relevant polynomials of age) into the model as a random effect, thus allowing the age-related trajectories to vary for each individual child. The age-related trends in the metabolic variables were not linear and therefore accounted for by polynomials of age. Age (continuous) was therefore centred on the mean age calculated across all annual time points (centred age $=0$ at 12.5 years) to aid the interpretation of such polynomials. Age was entered into the model as a fixed effect and as a random effect, then polynomials of increasing order were added one by one ( $\mathrm{age}^{2}, \mathrm{age}^{3}, \mathrm{age}^{4}$ and so on) again as both fixed and random effects. Random coefficients of the polynomials were retained provided that their variance components were positive. Fixed effects polynomials continued to be added until they were non-significant to ensure the best fitting age-related model [30]. Average physical activity (categorical or continuous) was then entered into the model as a main effect and as an interaction term with age and the polynomials of age. If one or more of these 'activity $\times$ age $^{\mathrm{p}}$, interaction terms were significant, a likelihood ratio test was performed to determine whether the inclusion of the interaction terms improved the overall fit of the model. If the interaction term was not significant, or not close to being significant $(p>0.10)$, the model was re-run without that interaction. 
The analysis was carried out for both sexes separately and combined and with and without controlling for any differences there might be in body fat percentage and pubertal status between the activity groups.

The distributions of insulin, IR and triacylglycerol were positively skewed in both sexes and were therefore logtransformed to ensure that the residuals did not violate the assumption of normality. The physical activity coefficient obtained when modelling these three variables was consequently back-transformed (with the exponential function) so that it represented the percentage difference in the outcome variable rather than the absolute difference on a logged scale. For all other metabolic variables, the activity coefficient represented the absolute difference. All analyses were performed using IBM SPSS version 19.0 (IBM, Armonk, NY, USA).

\section{Results}

Table 1 reports the characteristics of the cohort at baseline (age 9 years). Of note are the uniformity of age within and between the sexes, the higher body fat percentage and IR of the girls, their earlier attainment of peak height velocity and their lower levels of adolescent physical activity.

Most of the children included in this analysis were measured at most of the annual time points. Thus, $88 \%, 87 \%$ and $75 \%$, respectively, provided data on body fat percentage, IR and physical activity on at least six of the eight annual occasions from 9 years to 16 years. There were no significant differences for any of these three measures at 9 years between those who provided data on most occasions and those who did not (all $p>0.4$ ). Consequently, all cases were included in the analysis as multi-level modelling makes the most of the data available and retains participants who might otherwise be lost for lack of one or more data points.

\section{IR and glucose control according to physical activity}

Of the metabolic variables specific to glucose control (Table 2), neither fasting glucose nor $\mathrm{HbA}_{1 \mathrm{c}}$ were associated with physical activity, but fasting insulin and IR were, even after controlling for body fat $\%$ and pubertal status.

The age-related trend in IR for the entire cohort from 9-16 years followed an inverted U-shaped, quadratic curve (modelled by a second-order polynomial, age + age $^{2}$ ) with IR being at a similar level at ages 9 years and 16 years and peaking around 12-13 years. Figure 1 shows that the peak in IR was lower in the 'more active' group than the 'less active' group. In the unadjusted model, the 'activity group' main effect and the 'activity group $\times$ age $^{2}$, interaction were both statistically significant $(p<0.001$ and $p<0.01$ respectively, Table 2). The coefficient for the activity group main effect indicated that IR was $26.2 \%$ lower in the 'more active' group than in the 'less active' group at age 12.5 years (centred age $=0$ ) and the 'activity group $\times \mathrm{age}^{2}$ ' interaction coefficient indicated that this difference decreased by ' $1.5 \%$ multiplied by the number of years squared before or after age 12.5 years'e.g. at 14 years the difference was $-22.8 \%(-26.2 \%+$ $\left[1.5\right.$ years $\left.\left.{ }^{2} \times 1.5 \%\right]\right)$, at 15 years it was $-16.8 \%$ and at 16 years it was $-7.8 \%$ in the unadjusted model.

Given that the percentage of body fat was slightly, though consistently, higher in the less active than in the more active group throughout (by $\sim 4 \%$ in boys, $\sim 3 \%$ in girls, $p$ value ranging from $<0.01$ to 0.15 for all time points) and that body fat $\%$ is associated with IR ( $p<0.001$ at each time point), it was appropriate to enter body fat $\%$ into the model as a potential covariate. Although both measures of pubertal status tended to be similar between the activity groups, these were also entered as covariates because at least one of the measures (sometimes both) was associated with IR at each time point. The inclusion of these covariates only partly explained the age-varying effect of physical activity on IR. Results from the adjusted model indicated that IR was still significantly lower in the 'more active' group than in the 'less active' group at age 12.5 years (by $17.0 \%, p<0.001$, compared with $26.2 \%$ without covariates - body fat $\%$ reduced it by $\sim 7 \%$, pubertal stage by $\sim 2 \%$ ). They also showed that the difference still diminished by a function of age $^{2}$ either side of age 12.5 years ('activity group $\times \mathrm{age}^{2}$, interaction coefficient was $1.5 \%$, $p<0.01$; likelihood ratio test for inclusion of interaction terms with $\left.\chi^{2}(7,2), p=0.03\right)$. For example, by the ages of 14 years, 15 years and 16 years the difference had fallen to $-13.6 \%$, $-7.6 \%$ and $+1.4 \%$, respectively. For both the adjusted and unadjusted models the activity group difference in IR and insulin at age 12.5 years was slightly greater for girls than for boys, though this was not statistically significant ('activity group $\times$ age $^{2} \times$ sex' interaction, both $p>0.2$ ).

The findings obtained from the analysis of moderate-andvigorous activity as a continuous variable were, as expected, very similar to when it was analysed as a categorical variable. For example, for every extra $15 \mathrm{~min} /$ day spent in moderateand-vigorous activity, IR was $5.5 \%$ lower $(95 \% \mathrm{CI}-9.5 \%$, $-1.3 \%, p=0.01$ ) at age 12.5 years independent of body fat $\%$ and pubertal status. The 'activity $\times$ age $^{2}$, interaction term again reflected a reduction in the difference in IR either side of age 12.5 years (interaction coefficient $0.5 \%, 95 \%$ CI $0.1 \%$, $0.9 \%, p=0.02$ ) so by age 16 years the benefit had completely disappeared.

Fasting insulin correlates strongly with HOMA-IR in children $(r>0.9$ here) so, not surprisingly, the activity-related findings for insulin were almost identical to those found for IR.

\section{Lipids and BP according to physical activity}

Of the lipid variables reported in Table 3, only triacylglycerol in girls $(p=0.05)$, not boys $(p=0.51)$, was associated with 
Table 1 Participant characteristics (at age 9 years visit unless otherwise stated)

\begin{tabular}{|c|c|c|}
\hline Characteristic & Boys & Girls \\
\hline$N$ at 9 years visit & 151 & 149 \\
\hline$N$ at 10 years visit & 151 & 149 \\
\hline$N$ at 11 years visit & 151 & 143 \\
\hline$N$ at 12 years visit & 146 & 137 \\
\hline$N$ at 13 years visit & 145 & 136 \\
\hline$N$ at 14 years visit & 142 & 136 \\
\hline$N$ at 15 years visit & 141 & 136 \\
\hline$N$ at 16 years visit & 141 & 136 \\
\hline Age, years & $8.9(0.3)$ & $8.8(0.3)$ \\
\hline BMI, kg/m² & $17.1(2.6)$ & $18.1(2.9)$ \\
\hline Body fat, $\%$ & $18.6(8.7)$ & $25.7(8.1)$ \\
\hline Age at peak height velocity, years & $13.1(0.7)$ & $11.6(1.1)$ \\
\hline \multicolumn{3}{|l|}{ Pubic hair development, $\%(n / N)$} \\
\hline No pubic hair $(\mathrm{TS}=1)$ at 9 years & $97(142 / 147)$ & $93(136 / 147)$ \\
\hline Adult type hair $(\mathrm{TS} \geq 4)$ at 16 years & $94(132 / 140)$ & $83(113 / 136)$ \\
\hline \multicolumn{3}{|l|}{ Glucose control and IR } \\
\hline Glucose, mmol/1 & $4.8(0.4)$ & $4.7(0.3)$ \\
\hline Insulin, $\mathrm{pmol} / \mathrm{l}^{\mathrm{a}}$ & $26.4(16.0-38.9)$ & $35.4(25.7-49.3)$ \\
\hline HOMA-IR ${ }^{\mathrm{a}}$ & $0.54(0.33-0.81)$ & $0.75(0.53-1.02)$ \\
\hline $\mathrm{HbA}_{1 \mathrm{c}}, \%$ & $5.2(0.2)$ & $5.2(0.2)$ \\
\hline $\mathrm{HbA}_{1 \mathrm{c}}, \mathrm{mmol} / \mathrm{mol}$ & $33.8(2.4)$ & $33.4(2.2)$ \\
\hline \multicolumn{3}{|l|}{ Lipids and blood pressure } \\
\hline Triacylglycerol, mmol/ $\mathrm{l}^{\mathrm{a}}$ & $0.52(0.41-0.68)$ & $0.63(0.46-0.80)$ \\
\hline Total cholesterol, mmol/1 & $4.3(0.7)$ & $4.4(0.7)$ \\
\hline LDL-cholesterol, mmol/1 & $2.2(0.6)$ & $2.3(0.7)$ \\
\hline HDL-cholesterol, mmol/1 & $1.9(0.4)$ & $1.8(0.4)$ \\
\hline Cholesterol/HDL ratio & $2.4(0.5)$ & $2.6(0.6)$ \\
\hline $\mathrm{SBP}, \mathrm{mmHg}$ & $101(7)$ & $100(7)$ \\
\hline DBP, $\mathrm{mmHg}$ & $63(5)$ & $63(6)$ \\
\hline MAP & $75(5)$ & $75(6)$ \\
\hline \multicolumn{3}{|l|}{ Physical activity (mean 9 years to 16 years) } \\
\hline Moderate-and-vigorous physical activity (min/day) ${ }^{\mathrm{a}}$ & $50(40-65)$ & $35(28-45)$ \\
\hline
\end{tabular}

Data are presented as mean (SD) unless stated otherwise

${ }^{a}$ Median (interquartile range)

$\mathrm{TS}=$ Tanner stage physical activity independently of body fat $\%$ and pubertal status. The 'more active group' main effect coefficient in the adjusted model indicated that triacylglycerol was 9.7\% lower in the more active girls than in the less active girls across the entire age range. Findings were similar when activity was analysed as a continuous variable, although the difference was not statistically significant (in the adjusted model, triacylglycerol was $4.1 \%$ lower for every additional $15 \mathrm{~min} /$ day of moderate-and-vigorous activity, $p=0.08$ ).

The DBP of boys $(p=0.03)$ but not girls $(p=0.93)$ was associated with physical activity independently of body fat $\%$ and pubertal status. The 'more active group' main effect coefficient in the adjusted model indicated that DBP was
$1.20 \mathrm{mmHg}$ lower in the 'more active' boys than in the 'less active' boys across the entire age range. The corresponding finding derived from the analysis of physical activity as a continuous variable was similar, though the difference did not reach statistical significance (in the adjusted model, DBP was $0.41 \mathrm{mmHg}$ lower for every additional $15 \mathrm{~min} /$ day of moderate-and-vigorous activity, $p=0.11$ )

\section{Sensitivity analysis}

Re-running the multi-level models with total volume of physical activity as the main exposure variable produced very similar findings to those reported above for moderate-and- 


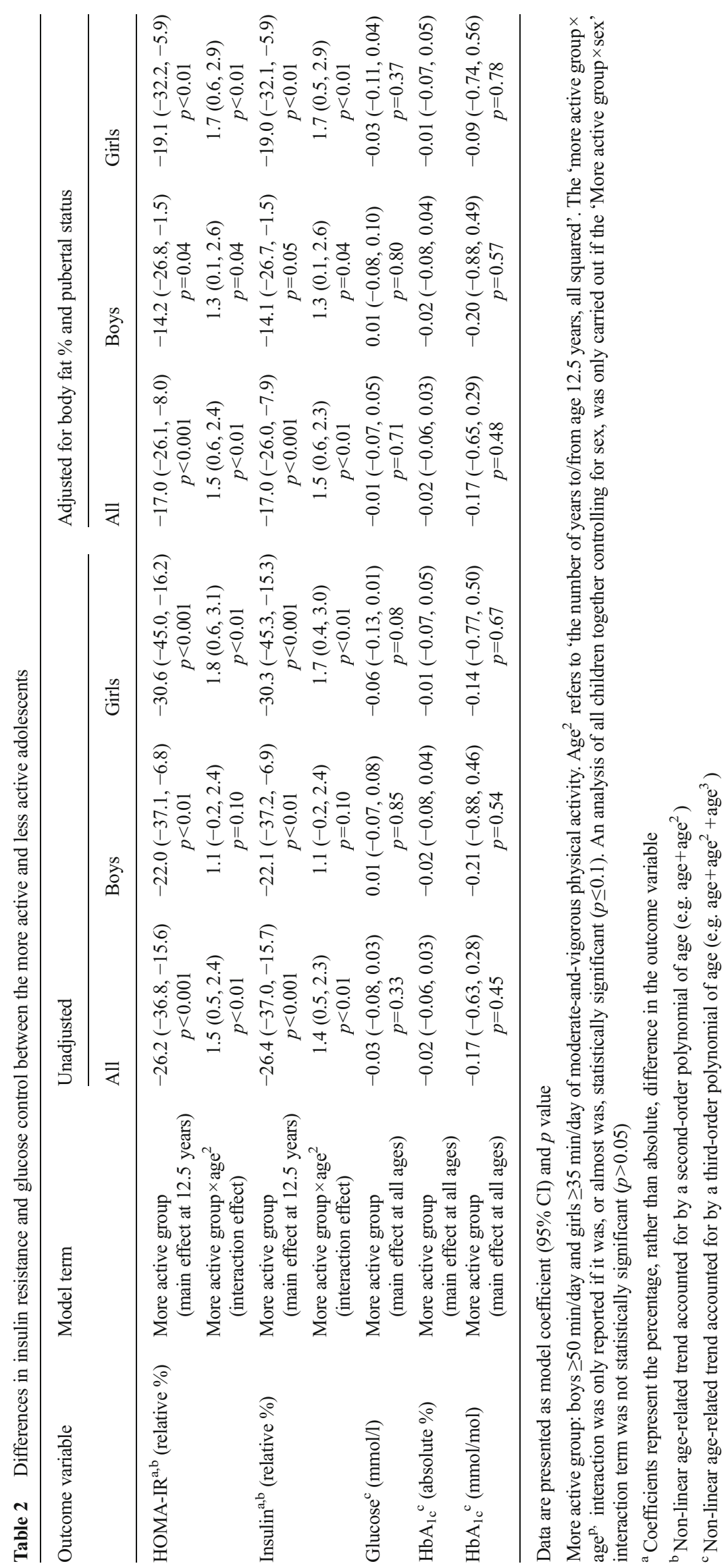




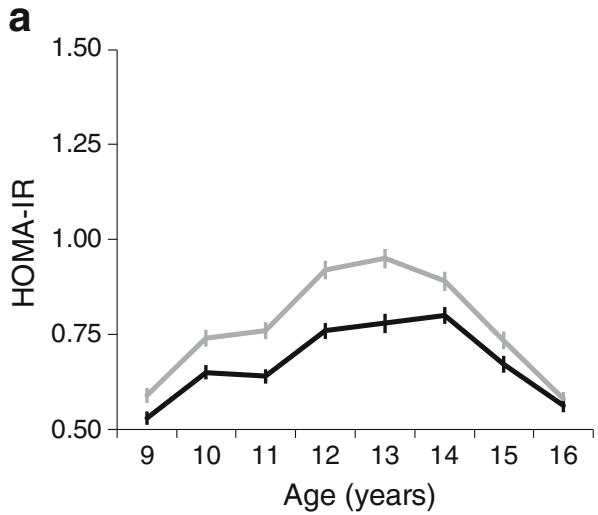

Fig. 1 Age-related changes in IR throughout adolescence, according to physical activity in boys (a) and girls (b). Black lines, more active group (time spent in moderate-and-vigorous physical activity above the median of 50 and $35 \mathrm{~min} /$ day for boys and girls, respectively); grey lines, less active group (below the median). Data points represent the back-

vigorous activity, although the magnitudes of the significant differences were marginally smaller. For example, when grouped by total volume of activity, IR was $14.9 \%$ lower $(p<0.001$, adjusted model $)$ at the age of 12.5 years in the more active group as compared with $17.0 \%$ when grouped by moderate-and-vigorous activity. However, the benefit for both had disappeared by the age of 16 years ('activity group $\times$ age $^{2}$, interaction: total activity $=1.3 \%, p=0.01$; moderate-andvigorous activity $=1.5 \%, p<0.01)$. When analysed for total volume of activity, triacylglycerol in girls was $6.5 \%$ lower $(p=0.27)$ and DBP in boys was $1.15 \mathrm{mmHg}$ lower $(p=0.04)$ in the more active group (compared with $9.7 \%$ and $1.20 \mathrm{mmHg}$, respectively, for moderate-and-vigorous activity).

\section{Discussion}

\section{Principal findings}

Physical activity attenuated the mid-adolescent peak in IR but the effect diminished progressively over the next few years and had disappeared completely by late adolescence. The temporary effect on IR was similar in both sexes and was independent of body fat and pubertal stage. Smaller, yet more permanent, benefits were seen in two other metabolic markers with triacylglycerol (in girls only) and DBP (in boys only) being marginally lower in the more physically active children throughout the whole of adolescence.

\section{Strengths and weaknesses}

Many studies now use accelerometers to measure physical activity objectively, though most rely on a single sampling period of 3-7 days, which we have shown previously is only

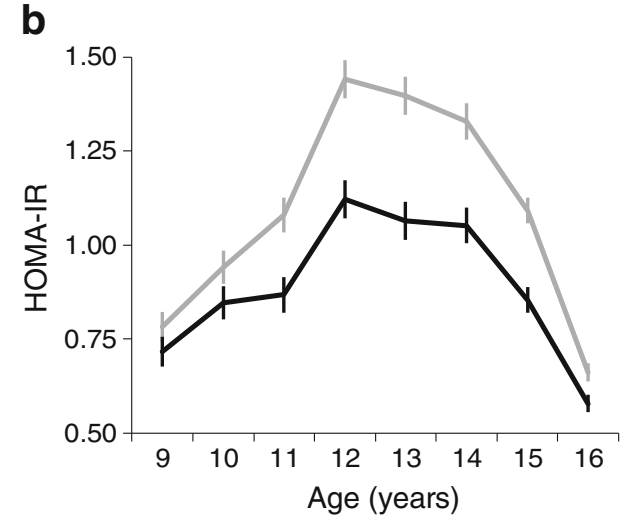

transformed age coefficients of logged IR for each activity group predicted by a longitudinal multi-level model (with age treated as a categorical variable for ease of presentation) unadjusted for covariates. Error bars represent the $95 \% \mathrm{CI}$ of these age-related coefficients

$\sim 70 \%$ reliable [31]. In this report, physical activity during adolescence was characterised with $95 \%$ reliability by averaging the eight annual measures of 7 day accelerometry. High reliability optimises the detection of even subtle relationships between long-term exposure to physical activity and changes in metabolic health. Fasting annual blood samples collected throughout adolescence in the same cohort enabled us to study trends in metabolic variables with high precision, and the uniform age of the cohort maximised resolution of time-related events. The study was nevertheless based on a single population of white adolescents living in the south-west of England. The homogeneity of race may have been helpful in revealing the findings we have reported but may limit the generalisability of the findings to other racial groups.

\section{Strengths and weaknesses in relation to other studies and any differences in their results}

Few longitudinal studies have examined IR and metabolic health in relation to objectively measured activity during adolescence and no studies have examined them in relation to the rise and fall of IR over that period. Telford and colleagues reported a longitudinal association between physical activity and IR from age 8 years to 12 years in boys $(p=0.04)$ [32], but did not find any cross-sectional association in the same boys at age 8 years or 10 years [33]. These findings are consistent with those of the current study as they imply that the relationship emerges as the boys approach mid-adolescence, when IR is at its highest. However, Telford et al did not make any measurements in children beyond the age of 12 years to confirm the late-adolescent fall in IR and the diminishing association with activity. Jago and colleagues reported a longitudinal association between physical activity and IR based on two points, at 9 years and 15 years [34], but, with limited time 


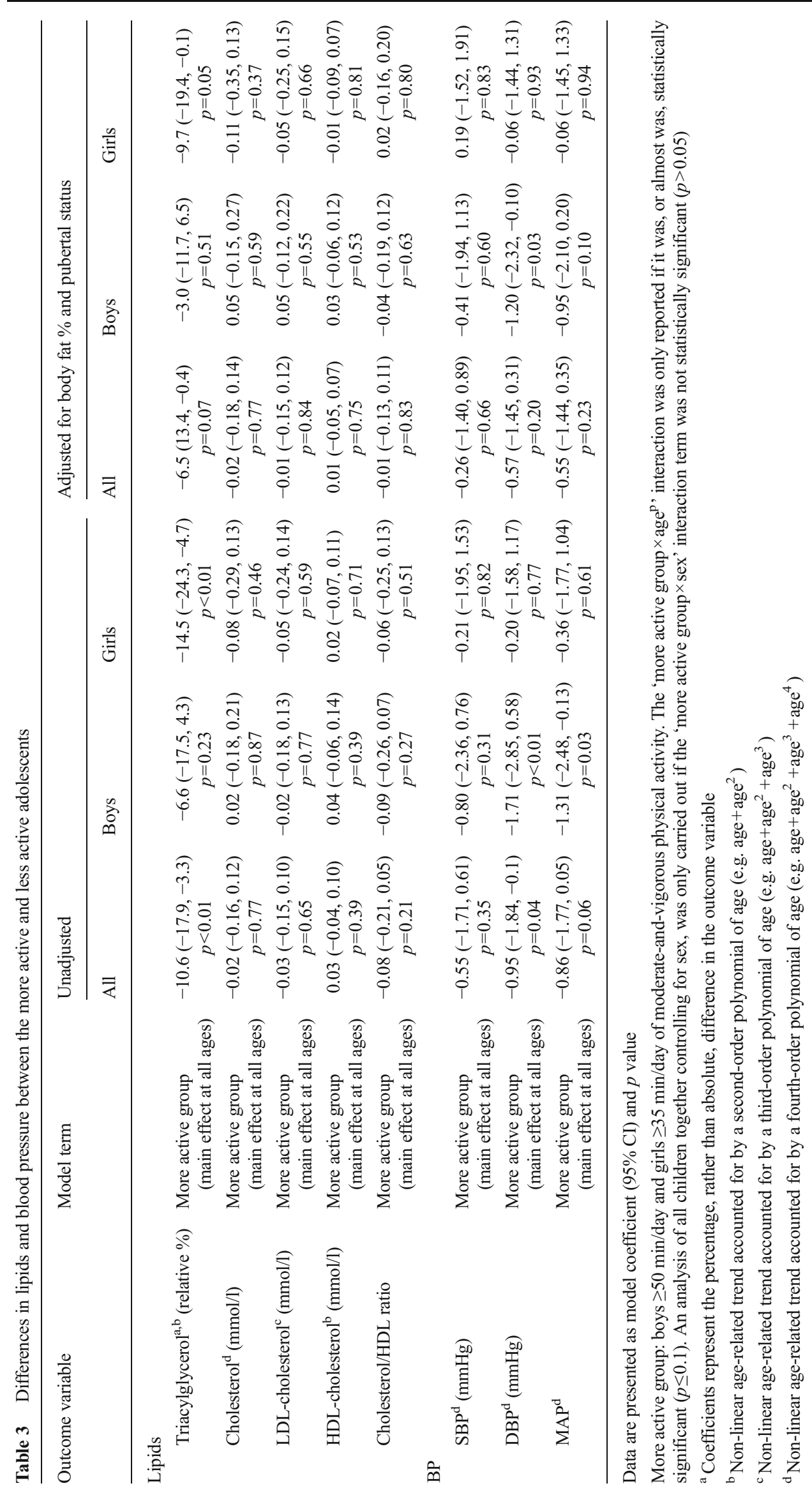


points, were not able to explore the age-related interaction found in the present study.

Most cross-sectional studies carried out in adolescents have incorporated a wide age range, making the interpretation of a single correlation difficult. A recent review reported that all nine cross-sectional studies using objective measures of physical activity found an (albeit small) inverse relationship between activity and IR ( $r \sim-0.2$ to -0.1$)$ [8]. While the single overall correlation produced by the present dataset $(9-16$ years $[r \sim-0.11])$ falls within this range, the age-specific correlations we found provided a strong indication that this association varied with age (e.g. for boys, $r=-0.17$ at 12 years and -0.02 at 16 years; for girls, $r=-0.31$ at 12 years and -0.03 at 16 years). More recently, a large cross-sectional analysis of some 5,000 children aged 4-18 years reported a significant inverse association between physical activity and fasting insulin $(\beta=-0.028$, $95 \%$ CI $-0.038,-0.017$ ) [35]. Unfortunately, the authors did not report age-specific associations, so it was unclear whether the strength of the association changed across the age range. A recent review of exercise-training trials did show that the impact on IR was slightly lower (though the difference was not statistically significant) at the later stages of puberty [9].

\section{Implications, unanswered questions and possible explanations}

These findings have implications for future interventions designed to improve the insulin sensitivity of children. An intervention that is deemed unsuccessful in 16-year-olds (when IR levels are low) may actually have led to significant reductions in IR had it been delivered to 12- to 13-year-olds (when IR levels were much higher). We do not know why IR should be attenuated by physical activity only in mid-adolescence. One possibility is that by the age of 16 years, when IR returns to its pre-adolescent levels [36], many children are once again sufficiently sensitive to the action of insulin to render any further change in IR, through increased activity, difficult to achieve. It is possible that the rise-and-fall cycle in IR during the course of adolescence reflects the 'hunting' behaviour that characterises a control loop under stress [37]. In such circumstances, the biological control loop would be expected to oscillate spontaneously between tissue function and diminishing beta cell reserve in order to defend blood glucose [38]. These oscillations could be more closely related to age than to puberty itself, as we have suggested previously [11], explaining the link to adolescence and limited interaction with pubertal stage. The higher insulin levels in the less active children during mid-adolescence will have put a greater demand on the beta cells over that period. It could be hypothesised that this greater demand on the beta cells during adolescence has a negative impact on the cells' function in adulthood, leading to an increased and/or accelerated risk of diabetes in later life.
We do not know why the improvements in triacylglycerol and DBP were sex specific or why similar improvements did not appear in the other lipid or BP measures. Nevertheless, we feel that the activity-related improvements in triacylglycerol (girls only) and DBP (boys only) demonstrate that the metabolic benefits of being more active are not simply restricted to IR.

\section{Conclusions}

Our findings may be important clinically, as physical activity appears to attenuate IR and insulin demand during midadolescence. It remains to be established whether the temporary attenuation in IR during mid-adolescence has implications for the development of diabetes in adolescence and adulthood, and for future metabolic health generally.

Acknowledgements The authors thank K. Brookes and V. Morgan (both of Plymouth Hospitals National Trust, UK) for their assistance with the collection of data.

Funding The EarlyBird study (BSM, JH, MM, ANJ, LDV, TJW) was supported by the Bright Future Trust, the Kirby Laing Foundation, the Peninsula Foundation and the EarlyBird Diabetes Trust. WEH was supported by the National Institute for Health Research (NIHR) Collaborations for Leadership in Applied Health Research and Care (CLAHRC). The funding bodies had no role in the study design, data collection and analysis or interpretation of results and no role in the writing of the report and the decision to submit the manuscript for publication.

Duality of interest The authors declare that there is no duality of interest associated with this manuscript.

Contribution statement TJW and LDV designed the study. BSM and TJW were involved in the conception of this particular piece of research. $\mathrm{BSM}, \mathrm{JH}, \mathrm{ANJ}$ and LDV were involved in the acquisition of the data and $\mathrm{BSM}, \mathrm{JH}, \mathrm{WEH}$ and MM were involved in the analysis and interpretation of the data. BSM and TJW drafted the manuscript, JH, ANJ, WEH, MM and LDV revised it and all authors approved the final version. TJW is responsible for the integrity of the work as a whole and is the guarantor of this work.

\section{References}

1. Health and Social Care Information Centre (2014) Statistics on obesity, physical activity and diet: England 2014. Available from www.hscic.gov.uk/pubs/sopad14, accessed 5 May 2015

2. Ogden CL, Carroll MD, Kit BK, Flegal KM (2014) Prevalence of childhood and adult obesity in the United States, 2011-2012. JAMA 311:806-814

3. Weiss R, Caprio S (2005) The metabolic consequences of childhood obesity. Best Pract Res Clin Endocrinol Metab 19:405-419

4. Ekelund U, Yngve A, Brage S, Westerterp K, Sjostrom M (2004) Body movement and physical activity energy expenditure in children and adolescents: how to adjust for differences in body size and age. Am J Clin Nutr 79:851-856 
5. Kamath CC, Vickers KS, Ehrlich A et al (2008) Clinical review: behavioral interventions to prevent childhood obesity: a systematic review and metaanalyses of randomized trials. J Clin Endocrinol Metab 93:4606-4615

6. Harris KC, Kuramoto LK, Schulzer M, Retallack JE (2009) Effect of school-based physical activity interventions on body mass index in children: a meta-analysis. CMAJ 180:719-726

7. Waters E, de Silva-Sanigorski A, Hall BJ et al (2011) Interventions for preventing obesity in children. Cochrane Database Syst Rev 12, CD001871. doi:10.1002/14651858.CD001871.pub3

8. Berman LJ, Weigensberg MJ, Spruijt-Metz D (2012) Physical activity is related to insulin sensitivity in children and adolescents, independent of adiposity: a review of the literature. Diabetes Metab Res Rev 28:395-408

9. Fedewa MV, Gist NH, Evans EM, Dishman RK (2014) Exercise and insulin resistance in youth: a meta-analysis. Pediatrics 133:e163-e174

10. Metcalf BS, Jeffery AN, Hosking J, Voss LD, Sattar N, Wilkin TJ (2009) Objectively measured physical activity and its association with adiponectin and other novel metabolic markers: a longitudinal study in children (EarlyBird 38). Diabetes Care 32:468-473

11. Jeffery AN, Metcalf BS, Hosking J, Streeter AJ, Voss LD, Wilkin TJ (2012) Age before stage: insulin resistance rises before the onset of puberty: a 9-year longitudinal study (EarlyBird 26). Diabetes Care 35:536-541

12. Kelly LA, Lane CJ, Weigensberg MJ, Toledo-Corral CM, Goran MI (2011) Pubertal changes of insulin sensitivity, acute insulin response, and beta-cell function in overweight Latino youth. J Pediatr 158:442-446

13. Amiel SA, Sherwin RS, Simonson DC, Lauritano AA, Tamborlane WV (1986) Impaired insulin action in puberty. A contributing factor to poor glycemic control in adolescents with diabetes. N Engl J Med 315:215-219

14. Fourlanos S, Narendran P, Byrnes GB, Colman PG, Harrison LC (2004) Insulin resistance is a risk factor for progression to type 1 diabetes. Diabetologia 47:1661-1667

15. Hojat M, Xu G (2004) A visitor's guide to effect sizes: statistical significance versus practical (clinical) importance of research findings. Adv Health Sci Educ Theory Pract 9:241-249

16. Office of National Statistics (2004) Ethnic group (UV09) neighbourhood statistics (Plymouth, UK). 2001 Census. Available from http://neighbourhood.statistics.gov.uk/dissemination/ LeadTableView.do? $\mathrm{a}=7 \& \mathrm{~b}=6275200 \& \mathrm{c}=$ plymouth $\& \mathrm{~d}=13 \& \mathrm{e}=$ $13 \& \mathrm{~g}=6390905 \& \mathrm{i}=1001 \times 1003 \times 1004 \& \mathrm{~m}=0 \& \mathrm{r}=1 \& \mathrm{~s}=$ 1431012531906\&enc $=1 \&$ dsFamilyId $=87$, accessed 5 May 2015

17. Voss LD, Kirkby J, Metcalf BS et al (2003) Preventable factors in childhood that lead to insulin resistance, diabetes mellitus and the metabolic syndrome: the EarlyBird diabetes study 1. J Pediatr Endocrinol Metab 16:1211-1224

18. Metcalf BS, Curnow JS, Evans C, Voss LD, Wilkin TJ (2002) Technical reliability of the CSA activity monitor: the EarlyBird Study. Med Sci Sports Exerc 34:1533-1537

19. Puyau MR, Adolph AL, Vohra FA, Butte NF (2002) Validation and calibration of physical activity monitors in children. Obes Res 10:150-157

20. Schmitz KH, Treuth M, Hannan P et al (2005) Predicting energy expenditure from accelerometry counts in adolescent girls. Med Sci Sports Exerc 37:155-161

21. Basterfield L, Adamson AJ, Pearce MS, Reilly JJ (2011) Stability of habitual physical activity and sedentary behavior monitoring by accelerometry in 6- to 8-year-olds. J Phys Act Health 8:543-547
22. Reilly JJ, Penpraze V, Hislop J, Davies G, Grant S, Paton JY (2008) Objective measurement of physical activity and sedentary behaviour: review with new data. Arch Dis Child 93:614-619

23. Department of Health (UK) (2011) Key principles. In: Fox K, Riddoch C (eds) Start active, stay active: a report on physical activity for health from the four home countries... Chief Medical Officers. Department of Health, UK, pp 16-19. Available from www.dh.gov.uk/prod_consum_dh/groups/dh_digitalassets/ documents/digitalasset/dh_128210.pdf, accessed 5 May 2015

24. Trost SG, Loprinzi PD, Moore R, Pfeiffer KA (2011) Comparison of accelerometer cut points for predicting activity intensity in youth. Med Sci Sports Exerc 43:1360-1368

25. Thorsen S (2014) Weather in UK-England-Plymouth, time and date. Available from www.timeanddate.com/weather/uk/ plymouth, accessed 5 May 2015

26. Cliff DP, Reilly JJ, Okely AD (2009) Methodological considerations in using accelerometers to assess habitual physical activity in children aged 0-5 years. J Sci Med Sport 12:557-567

27. Mattocks C, Ness A, Deere K et al (2008) Early life determinants of physical activity in 11 to 12 year olds: cohort study. BMJ 336:26-29

28. Levy JC, Matthews DR, Hermans MP (1998) Correct homeostasis model assessment (HOMA) evaluation uses the computer program. Diabetes Care 21:2191-2192

29. Gungor N, Saad R, Janosky J, Arslanian S (2004) Validation of surrogate estimates of insulin sensitivity and insulin secretion in children and adolescents. J Pediatr 144:47-55

30. Brown H, Prescott R (2006) Repeated measures data. In: Brown H, Prescott R (eds) Applied mixed models in medicine, 2nd edn. Wiley, Chichester, pp 215-270

31. Metcalf BS, Voss LD, Hosking J, Jeffery AN, Wilkin TJ (2008) Physical activity at the government-recommended level and obesity-related health outcomes: a longitudinal study (Early Bird 37). Arch Dis Child 93:772-777

32. Telford RD, Cunningham RB, Telford RM et al (2012) Effects of changes in adiposity and physical activity on preadolescent insulin resistance: the Australian LOOK longitudinal study. PLoS One 7, e47438

33. Telford RD, Cunningham RB, Shaw JE et al (2009) Contrasting longitudinal and cross-sectional relationships between insulin resistance and percentage of body fat, fitness, and physical activity in children-the LOOK study. Pediatr Diabetes 10:500-507

34. Jago R, Wedderkopp N, Kristensen PL et al (2008) Six-year change in youth physical activity and effect on fasting insulin and HOMA-IR. Am J Prev Med 35:554-560

35. Ekelund U, Luan J, Sherar LB, Esliger DW, Griew P, Cooper A (2012) Moderate to vigorous physical activity and sedentary time and cardiometabolic risk factors in children and adolescents. JAMA 307:704-712

36. Higgins J, Dohahoo WT (2008) Adiposity is the enemy: body composition and insulin sensitivity. In: Zeitler PS, Nadeau KJ (eds) Insulin resistance: childhood precursors and adult disease. Humana Press/Springer, New York, pp 125-148

37. Hosking J, Metcalf BS, Jeffery AN, Streeter AJ, Voss LD, Wilkin TJ (2013) Evidence of early beta-cell deficiency among children who show impaired fasting glucose: 10-yr cohort study (EarlyBird 56). Pediatr Diabetes 14:481-489

38. Wilkin TJ (1998) Endocrine feedback control in health and disease. In: Bittar EE, Bittar N (eds) The principles of medical biology. JAI Press, Greenwich, pp 1-28 